\title{
Nonadiabatic chemical-to-electrical energy conversion in heterojunction nanostructures
}

\author{
Eduard G. Karpov* \\ Department of Civil \& Materials Engineering, University of Illinois, Chicago, Illinois 60607, USA \\ Ievgen Nedrygailov \\ Fachbereich Chemie, Universität Duisburg-Essen, D-45117 Essen, Germany \\ (Received 19 March 2010; published 27 May 2010)
}

\begin{abstract}
Nonadiabatic energy dissipation by electron subsystem of nanostructured solids unveil interesting opportunities for the solid-state energy conversion and sensor applications. We found that planar $\mathrm{Pt} / \mathrm{GaP}$ and $\mathrm{Pd} / \mathrm{GaP}$ Schottky structures with nanometer thickness metallization demonstrates a nonadiabatic channel for the conversion into electricity the energy of a catalytic hydrogen-to-water oxidation process on the metal layer surface. The observed above thermal current greatly complements the usual thermionic emission current and its magnitude is linearly proportional to the rate of formation and desorption of product water molecules from the nanostructure surface. The possibilities of utilizing the nonadiabatic functionality in chemical-to-electrical energy conversion devices are discussed.
\end{abstract}

DOI: 10.1103/PhysRevB.81.205443

PACS number(s): 84.60. - h, 73.21.- b, 73.23.Ad, 84.90.+a

\section{INTRODUCTION}

Many chemical processes on metal surfaces flow highly exothermally providing energies in the order of electron volts per reaction event. ${ }^{1,2}$ The successive processes of energy dissipation have until recently been interpreted within the thermal equilibrium settings implying a dominant role of the adiabatic energy exchange with the reactive adsorbate species and leading simply to heating of the metal surface. Meanwhile, more recent studies indicate that the chemical energy dissipation may also occur through direct excitation of the electronic system of the metal, i.e., in a thermodynamically nonadiabatic manner. For example, the publications $^{3-5}$ show involvement of the nonadiabatic mechanism of energy dissipation during chemisorption of monatomic and polyatomic species, such as $\mathrm{H}, \mathrm{O}, \mathrm{Xe}, \mathrm{NO}$, and $\mathrm{C}_{2} \mathrm{H}_{4}$, to the ultrathin $\mathrm{Ag}$ films deposited on top of $n$ and $p$-type $\mathrm{Si}$ substrates and maintained at $135 \mathrm{~K}$ during the experiments. The metal-semiconductor heterostructures (Schottky diodes) Ag-Si were designed in a way allowing for the detection of the chemically induced electron excitations in the metal as a hot charge carriers current across the structure. These charge carriers are generated nonadiabatically in the exoergic chemical events on the surface and then emitted ballistically over the Schottky barrier and into the underlying semiconductor wafer. Chemically induced excitation of hot electron was also demonstrated in $\mathrm{TiO}_{2}-$ and $\mathrm{GaN}$-based Schottky nanodiodes at elevated sample temperatures, sufficient to initiate the heterogeneous catalytic processes of carbon monoxide oxidation. ${ }^{6-8}$

The present authors ${ }^{9,10}$ utilized $\mathrm{Pd} / 6 \mathrm{H}-\mathrm{SiC}$ Schottky heterostructures with nanometer thickness $\mathrm{Pd}$ films to demonstrate an effective chemically induced excitation of hot electrons at temperatures $350-800 \mathrm{~K}$; the higher temperature regime allowed using the more complex reaction of catalytic hydrogen-to-water oxidation on the Pd film surface for the nonadiabatic electron excitation in the same metal film. Another group also used the catalytic hydrogen oxidation in a lower temperature range of $298-373 \mathrm{~K}$ to demonstrate hotelectron generation in $\mathrm{Pt} / \mathrm{TiO}_{2}$ Schottky nanostructures. ${ }^{11}$
The study of nonadiabatic energy dissipative processes in the course of exoergic chemical reactions on metal surfaces provides interesting fundamental insights ${ }^{6,12,13}$ and also unveils exciting practical opportunities. One possibility being under consideration presently is the development of a distinct class of chemical-to-electrical energy conversion devices, ${ }^{9,14}$ which would utilize the nonadiabatic process to minimize heat losses and provide superior efficiency and energy density compared to the existing fuel cells. However, the relevant energy exchange processes arising form the reactive gas/metal surface interactions and leading to the chemically induced excitation of energetic electrons on metal surfaces without resorting to heat (as during photoexcitation of electrons in photovoltaic devices) are insufficiently understood at the present time. ${ }^{3,12,15}$ Besides, the quantum efficiency, number of electron generated per reaction event, of the systems studied by this time is relatively low being in the range of $10^{-4}-10^{-3}$. Therefore, elucidation of basic properties this process for various metal-semiconductor heterostructures is very important for the development of nonadiabatic chemical-to-electrical energy conversion devices.

This paper provides a discussion of the direct conversion to electricity of chemical energy released in the hydrogen-towater oxidation process on the surface of thin-film metalsemiconductor nanostructures with Schottky barriers using the wide band gap $n-\mathrm{GaP}\left(E_{g}=2.3 \mathrm{eV}\right)$. For the comparison and study of the influence of Schottky contact physical properties on the energy conversion efficiency, the Pd and Pt thin films are considered. Temporal behavior of the generated current and its linearity to water desorption from the device surface are discussed. Individual contributions of the adiabatic and nonadiabatic channels of energy dissipation are estimated.

\section{BASIC MECHANISMS}

The basic concept of chemically induced charge-carriers detection utilized in the present work is schematically shown in Fig. 1. The thin-film metallization of the Schottky junction 


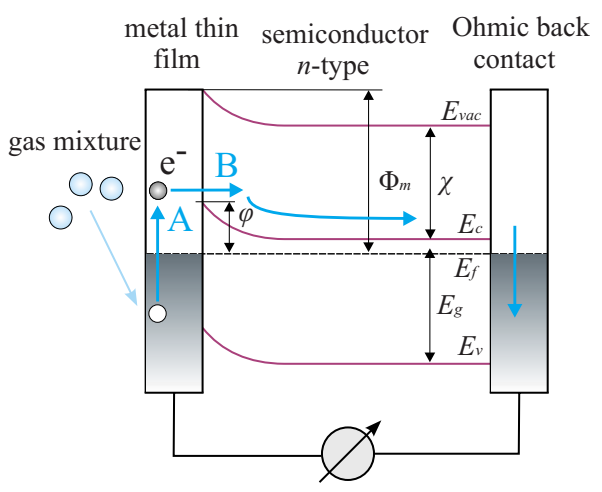

FIG. 1. (Color online) Detection of chemically induced current in a metal-semiconductor heterojunction structure with a catalytic metal nanofilm; $\phi$ is the Schottky barrier height, $E_{v a c}$ and $E_{f}$-vacuum and Fermi levels of electrons, $E_{c}$ - conduction-band bottom, $E_{v}$-valence-band top, $\chi$ - electron affinity, and $\Phi_{m}$-metal work function. Energy released from the $2 \mathrm{H}_{2}+\mathrm{O}_{2} \rightarrow 2 \mathrm{H}_{2} \mathrm{O}$ process on the film surface can dissipate adiabatically generating heat, as well as nonadiabatically leading to the direct excitation of energetic (above thermal) electrons in the metal film. These electrons have energies and lifetimes sufficient to travel ballistically over the Schottky barrier and toward the semiconductor region. They further drift into the external circuit via the Ohmic back contact.

also serves as catalyst to the hydrogen oxidation in the lowpressure reactive oxyhydrogen mixture admitted to the film surface. Other reactive mixtures may alternatively be used, however, the $2 \mathrm{H}_{2}+\mathrm{O}_{2} \rightarrow 2 \mathrm{H}_{2} \mathrm{O}$ process employed here is very interesting for the sustainable energy perspectives. The energetic effects of surface recombination of hydrogen derived radicals are $1.17 \mathrm{eV}$ and $0.91 \mathrm{eV}$ for the $\mathrm{H}+\mathrm{OH}$ $\rightarrow \mathrm{H}_{2} \mathrm{O}$ and $\mathrm{OH}+\mathrm{OH} \rightarrow \mathrm{O}+\mathrm{H}_{2} \mathrm{O}$ processes, respectively. ${ }^{15-17}$ These two reactions comprise the last step in the catalytic oxidation of hydrogen to water on metal surfaces. ${ }^{15,17}$ The energy released in these processes may dissipate nonadiabatically via the direct excitation of electrons with energies 1 $\mathrm{eV}$ above the Fermi level in the metal film. The mean-free path of such electrons is in the range of $8-30 \mathrm{~nm}$ for most catalytic metals, ${ }^{18}$ and therefore they can travel across sufficiently thin films toward the device interface without significant attenuation of the current density. Next, if the energy of these chemically excited energetic electrons is greater than the Schottky barrier height at the metal $/ n$-type semiconductor interface, they will participate in the emission over the barrier and toward the semiconductor substrate. This process is above thermal being similar to the photoinduced emission in photovoltaic devices. It is manifested by the "chemicurrent" registered in the external circuit of the device. The surface chemical reaction also leads to the usual heating of the metal film, and therefore to an adiabatic thermal contribution to the external circuit current as well. This "thermocurrent" arises from the thermionic emission across the Schottky barrier, ${ }^{9}$ as well as from the thermoelectric Thomson effect ${ }^{19}$ associated with electron diffusion against the temperature gradient in the semiconductor layer. Compared to the thermionic emission, ${ }^{20-22}$ the thermoelectric component was shown negligible in the recent chemicurrent studies of Schottky nanostructures. ${ }^{23}$ The total chemically induced current is

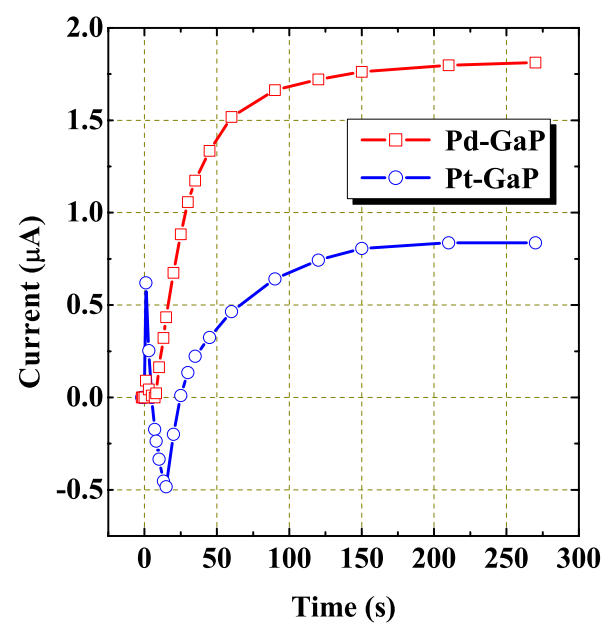

FIG. 2. (Color online) Kinetics of the total chemically induced current measured during exposure of $\mathrm{Pd} / \mathrm{GaP}$ and $\mathrm{Pt} / \mathrm{GaP}$ samples at temperature $660 \mathrm{~K}$ to the oxyhydrogen stoichiometric mixture at pressure 0.2 Torr.

then a sum of the chemicurrent and thermocurrent, $I_{\text {total }}$ $=I_{\mathrm{ch}}+I_{\mathrm{th}}$.

\section{RESULTS AND DISCUSSION}

The metal-semiconductor heterojunction nanostructures with Schottky barriers were manufactured by depositing continuous $15 \mathrm{~nm} \mathrm{Pt}$ and Pd layers onto a polished side of $n$-type $\mathrm{GaP}$ substrates of the size $18 \times 18 \times 0.5 \mathrm{~mm}^{3}$ and resistivity $0.03 \Omega \mathrm{cm}$, using e-beam evaporation in high vacuum $\left(<10^{-7}\right.$ Torr) at the rate $0.15 \AA /$ s and substrate temperature $370 \mathrm{~K}$. A partial masking of the substrate was applied to obtain square centered Schottky contacts of surface area $23 \mathrm{~mm}^{2}$. Ohmic contacts were applied to the entire reverse nonpolished side of the substrates by thermal infusion of pure indium at $500 \mathrm{~K}$. The fabricated structures had nonlinear diode-type voltage current characteristics. Thermionic emission analysis ${ }^{24}$ of the voltage-current curves was employed to determine the effective Schottky barrier height: $0.77 \mathrm{eV}(\mathrm{Pd} / \mathrm{GaP})$ and $0.74 \mathrm{eV}(\mathrm{Pt} / \mathrm{GaP})$. The chemically induced current was measured with a $10 \mathrm{k} \Omega$ input impedance nanoammeter.

Kinetics of the current recorded continuously during exposure of the $\mathrm{Pd} / \mathrm{GaP}$ and $\mathrm{Pt} / \mathrm{GaP}$ samples to the $2 \mathrm{H}_{2}+\mathrm{O}_{2}$ mixture at pressure 0.2 Torr are shown in Fig. 2. A sharp peak of the current magnitude is observed at the time of mixture admission followed by an exponential decay toward the negative region and a successive steady growth toward a stationary value. The stationary regimes were typically achieved in 2-3 min after mixture admission. The magnitude of the stationary current increases with sample surface temperature reaching the values $1.8 \mu \mathrm{A}$ and $0.84 \mu \mathrm{A}$, respectively, for the $\mathrm{Pd} / \mathrm{GaP}$ and $\mathrm{Pt} / \mathrm{GaP}$ structures at $660 \mathrm{~K}$, see Fig. 3. Here, a decomposition of the current into the nonadiabatic (chemi) and adiabatic (thermo) components is also provided. Separation and measurement of the thermal component were accomplished by externally heating the given structures in vacuum, i.e., without mixture admission, to the 


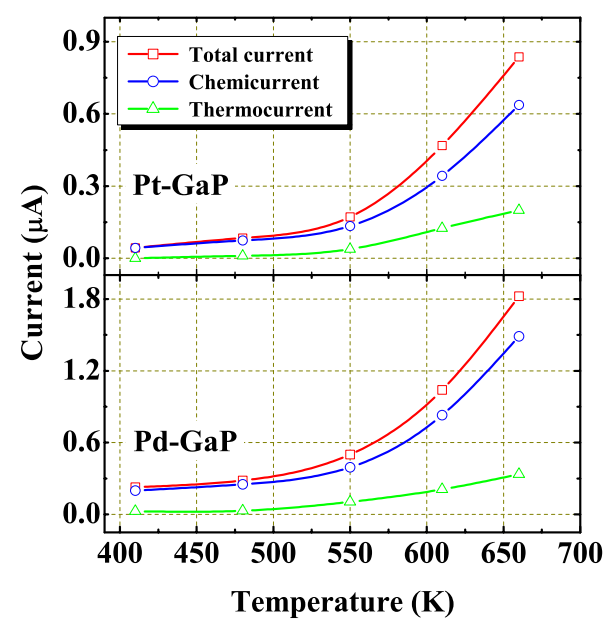

FIG. 3. (Color online) Decomposition of the total current generated in $\mathrm{Pd} / \mathrm{GaP}$ and $\mathrm{Pt} / \mathrm{GaP}$ structures into the nonadiabatic (chemi) and adiabatic (thermo) components. The thermal current is owed to the usual thermionic emission over the Schottky barrier and to the thermoelectric Thompson effect in the semiconductor.

same recorded temperature points. For this purpose, two thermocouples were attached to the bottom and top surfaces of the sample and their signals were recorded together with the chemicurrent kinetics. Catalytic oxidation of hydrogen on the sample surface led to a small increase in the surface temperature relative to the sample working temperature. The working temperature was maintained constant with the substrate heater at bottom side of the sample. A temperature gradient was arising across the sample as a result of this additional temperature increase and this gradient was dependent on the working temperature. In the next step, the same temperatures were reproduced on the sample sides using an electric heater instead of the chemical reaction while the working chamber was evacuated to high vacuum. The current generated in these settings was viewed the thermal component of the total current. The nonadiabatic component was then determined by subtracting the thermal current from the total measured current. This analysis showed the nonadiabatic component to comprise $75-80 \%$ of the total current at $660 \mathrm{~K}$ and this fraction may further grow with temperature.

The chemicurrent kinetics shown in Fig. 2 reflect physical processes on the sample surface associated with the catalytic oxidation of hydrogen to water. The sharp maxima of the current at initial times are related to the adsorption of molecular hydrogen and oxygen onto the Pd or Pt nanofilm, whose surface is yet pristine. Upon saturation of the surface with hydrogen and oxygen molecules the current drops to the zero level and below due to the charge-transfer processes between the solid surface and gas adsorbate. ${ }^{15,25}$ The successive gradual increase in the chemicurrent toward the stationary values is a result of two competing processes: recombination of $\mathrm{H}, \mathrm{O}$, and $\mathrm{OH}$ radicals appearing on the surface from the dissociation of hydrogen and oxygen molecules ${ }^{26-28}$ and accumulation of water adsorbate, and desorption of the product water molecules to the gas phase.

For a constant pressure of the oxyhydrogen mixture, the stationary current magnitude is determined by the rate of formation and desorption of water molecules from the

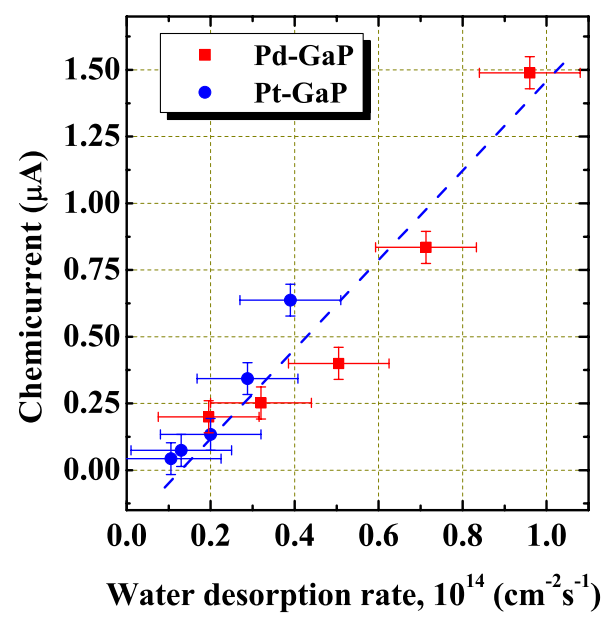

FIG. 4. (Color online) Chemicurrent as a function of water molecules desorption rate, $r_{w}$. The chemicurrent magnitude can be approximated by a linear relationship of the type $I_{\mathrm{ch}}=e \eta r_{w} S$, where $e$ - elementary charge, $S$-nanofilm surface area, and $\eta$ is a dimensionless efficiency constant.

sample surface. Therefore, the growing behavior of the chemicurrent in Fig. 3 plots can be explained by the increase in $\mathrm{H}_{2} \mathrm{O}$ desorption rates at elevated temperatures. This viewpoint also explains the greater magnitudes of the chemicurrent observed for the $\mathrm{Pd} / \mathrm{GaP}$ sample compared to the $\mathrm{Pt} / \mathrm{GaP}$ sample current. Indeed, analysis of the mass-spectrometry data gives the flux of the desorbing water molecules $r_{w}$ being $1.01 \times 10^{14} \mathrm{~cm}^{-2} \mathrm{~s}^{-1}$ and $0.42 \times 10^{14} \mathrm{~cm}^{-2} \mathrm{~s}^{-1}$ for the $\mathrm{Pd} /$ $\mathrm{GaP}$ and $\mathrm{Pt} / \mathrm{GaP}$ nanostructures, respectively. Meanwhile, the relative magnitudes of the chemicurrents and $\mathrm{H}_{2} \mathrm{O}$ fluxes for the palladium- and platinum-type structures are very close to each other, $I_{\mathrm{ch}}^{(\mathrm{Pd})} / I_{\mathrm{ch}}^{(\mathrm{Pt})} \approx r_{w}^{(\mathrm{Pd})} / r_{w}^{(\mathrm{Pt})} \approx 2.4$. These results can be summarized by writing the chemicurrent in the form $I_{\mathrm{ch}}$ $=e \eta r_{w} S$, where $e$ is the elementary charge, $S$ surface area of $\mathrm{Pd}$ and Pt films, and the dimensionless $\eta$ is a fundamental quantum efficiency parameter of the studied sample. The physical meaning of $\eta$ is the number of electrons, excited nonadiabatically and able to overcome the Schottky barrier, per one water molecule formed on and desorbed from the nanofilm surface. This constant is a basic efficiency parameter of the nonadiabatic energy conversion process. An experimental verification of the expression $I_{\mathrm{ch}}=e \eta r_{w} S$ is provided in Fig. 4, where the measurement data from both $\mathrm{Pd} /$ $\mathrm{GaP}$ and $\mathrm{Pt} / \mathrm{GaP}$ samples are well approximated by a linear dependence. The value of the coefficient $\eta$ found as the slope of Fig. 4 plot is about 0.1 , which corresponds to the excitation of one hot electron in the metal nanofilm per ten water molecules formed on the surface. The linear behavior observed in Fig. 4 plot suggests freedom of selection of the chemical reaction type and materials for the nanostructure fabrication; the reaction is only required to have all desorptive products and an exoergic stage with energy yield greater than the Schottky barrier height, and the nanostructure anode material has be catalytic to the reaction chosen.

\section{CONCLUSION}

We conclude that planar $\mathrm{Pd} / \mathrm{GaP}$ and $\mathrm{Pt} / \mathrm{GaP}$ Schottky structures with nanometer thickness metallization can dem- 
onstrate a strong nonadiabatic channel of surface chemical energy dissipation. The nonadiabatic component comprises up to $80 \%$ of the total chemically induced electric current arising from the catalytic oxidation of hydrogen to water on the nanostructure surface. The magnitude of the stationary current is determined by the rate of formation and desorption of product water molecules and well approximated by a linear dependence. The value of $\eta=0.1$ found here is remarkably higher than typical quantum efficiencies of the chemically induced emission of electrons into vacuum being in the range of $10^{-6}-10^{-8}$ electrons per chemical event on metal surface. ${ }^{29}$ This result provides grounds to seriously consider the chemically induced electron excitation in metals not only for studying gas-surface inelastic interactions, but also for energy conversion applications; these would combine physi- cal functionality of the photovoltaic cells with usual advantages of the hydrogen-based power, such as cleanness, mobility, and independence of external conditions. The energy efficiency estimated as ratio of the input chemical and output electric powers is not higher than a few percents for the samples studied here. Nonetheless, since the maximal theoretical efficiency of single junction barrier-layer structures as energy conversion devices is about $41 \%,{ }^{30}$ a quest for more efficient metal-semiconductor combinations and device architectures will be well worthy of the effort. In particular, incorporation of a nanodot pattern to the device nanocathode could increase the catalyst surface area, as well as density of the adsorption centers, and thus increase the reaction turnover rate, leading to higher conversion efficiency and volumetric power density of these devices. *ekarpov@uic.edu

${ }^{1}$ E. Shustorovich, Metal-Surface Reaction Energetics (VCH, Weinheim, 1991).

${ }^{2}$ N. Selley, Chemical Energetics (Arnold, London, 1971).

${ }^{3}$ B. Gergen, H. Nienhaus, W. Weinberg, and E. McFarland, Science 294, 2521 (2001).

${ }^{4}$ H. Nienhaus, H. S. Bergh, B. Gergen, A. Majumdar, W. H. Weinberg, and E. W. McFarland, Phys. Rev. Lett. 82, 446 (1999).

${ }^{5}$ H. Nienhaus, H. Bergh, B. Gergen, A. Majumdar, W. Weinberg, and E. McFarland, Appl. Phys. Lett. 74, 4046 (1999).

${ }^{6}$ J. Park, J. Renzas, A. Contreras, and G. Somorjai, Top. Catal. 46, 217 (2007).

${ }^{7}$ J. Park, J. Renzas, B. Hsu, and G. Somorjai, J. Phys. Chem. C 111, 15331 (2007).

${ }^{8}$ J. Park, H. Lee, J. Renzas, Y. Zhang, and G. Somorjai, Nano Lett. 8, 2388 (2008)

${ }^{9}$ E. Karpov and I. Nedrygailov, Appl. Phys. Lett. 94, 214101 (2009).

${ }^{10}$ E. Karpov and I. Nedrygailov, Virtual J. Nanoscale Sci. Technol. 19 (24) (2009).

${ }^{11}$ A. Hervier, J. R. Renzas, J. Y. Park, and G. Somorjai, Nano Lett. 9, 3930 (2009).

${ }^{12}$ H. Nienhaus, Surf. Sci. Rep. 45, 1 (2002).

${ }^{13}$ A. Wodtke, J. Tully, and D. Auerbach, Int. Rev. Phys. Chem. 23, 513 (2004)

${ }^{14}$ E. Karpov and I. Nedrygailov, Proceedings of ASME Third International Conference on Energy Sustainability, San Francisco,
CA, 2009, CD-ROM, Paper No. ES2009-90111.

${ }^{15}$ E. Hasselbrink, Surf. Sci. 603, 1564 (2009).

${ }^{16}$ W. Williams, C. Marks, and L. Schmidt, J. Phys. Chem. 96, 5922 (1992).

${ }^{17}$ V. Kwasniewski and L. Schmidt, J. Phys. Chem. 96, 5931 (1992).

${ }^{18}$ K. Frese and C. Chen, J. Electrochem. Soc. 139, 3234 (1992).

${ }^{19}$ C. Kittel, Introduction to Solid State Physics, 8th ed. (Wiley, New York, 2005).

${ }^{20}$ A. Shakouri and J. E. Bowers, Appl. Phys. Lett. 71, 1234 (1997).

${ }^{21}$ G. Zeng et al., Appl. Phys. Lett. 88, 113502 (2006).

${ }^{22}$ L. Whitlow and T. Hirano, J. Appl. Phys. 78, 5460 (1995).

${ }^{23}$ U. Hagemann, D. Krix, and H. Nienhaus, Phys. Rev. Lett. 104, 028301 (2010).

${ }^{24}$ W. Monch, Electronic Properties of Semiconductor Interfaces (Springer, Berlin, 2004).

${ }^{25}$ M. Mizielinski, D. Bird, M. Persson, and S. Holloway, Surf. Sci. 602, 2617 (2008)

${ }^{26}$ J. Fogelberg and L. Ekedahl, Surf. Sci. 350, 91 (1996).

${ }^{27}$ B. Hellsing, B. Kasemo, and V. Zhdanov, J. Catal. 132, 210 (1991).

${ }^{28}$ M. Löfdahl, M. Eriksson, M. Johansson, and I. Lundström, J. Appl. Phys. 91, 4275 (2002).

${ }^{29}$ T. Greber, Surf. Sci. Rep. 28, 1 (1997).

${ }^{30}$ M. A. Green, Third Generation Photovoltaics (Springer, Berlin, 2006). 